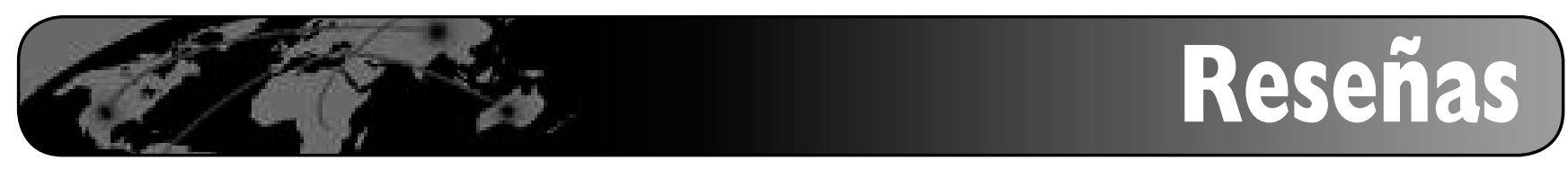

\title{
31th ELAG Seminar sobre la biblioteca 2.0
}

\author{
Por Sílvia Redondo
}

Redondo, Sílvia. "31th ELAG Seminar sobre la biblioteca 2.0". En: El profesional de la información, 2007, noviembre-diciembre, v. 16, n. 6, pp. 657-660.

DOI: 10.3145/epi.2007.nov.13

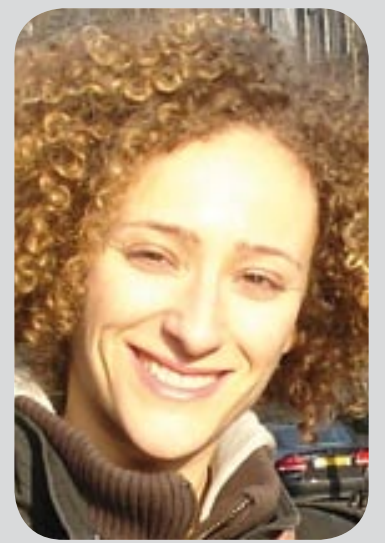

Sílvia Redondo Iniesta es licenciada en historia por la Universitat de Barcelona y en documentación por la misma universidad, donde también cursa el doctorado y ha obtenido el Diploma de Estudios Avanzados. Es bibliotecaria del CRAl de la Universitat de Barcelona y profesora asociada de la Facultat de Biblioteconomia i Documentació de dicha universidad.

\section{Introducción}

EL 3ITH ELAG LIBRARY SYSTEMS SEMINAR tuvo lugar en Barcelona entre los días 9 y 11 de mayo, y reunió a 120 participantes vinculados al mundo de las bibliotecas y las tecnologías de la información y la comunicación (TIC) de más de 20 países europeos.

Este evento anual fue organizado en esta ocasión por el Consorci de Biblioteques de les Universitats Catalanes (CBUC), la Universitat de Barcelona (UB), la Universitat Autònoma de Barcelona $(U A B)$ y la Universitat Pompeu Fabra (UPF) y se desarrolló en el Edificio Histórico de la $U B$ y en la recién in- augurada Biblioteca de Filosofía, Geografía e Historia de la misma universidad.

El ELAG Seminar ha tenido como lema central de esta edición la Library 2.0 (biblioteca 2.0), aunque también se trataron otros aspectos como el open access, las publicaciones digitales o la interoperabilidad entre sistemas. El seminario se estructuró en tres bloques a lo largo de los tres días: los papers, los workshops y los progress reports, que se hicieron el último día.

Los papers son conferencias en donde pueden asistir todos los participantes. Con el fin de dar a conocer el ámbito bibliotecario de cada país en donde se realiza el seminario, se combinan intervencio- nes de carácter local con otras más generales relacionadas con el lema principal del evento. Por su parte, los workshops son talleres o grupos de trabajo coordinados por un especialista y destinados a fomentar la comunicación, el intercambio de ideas y la participación de los asistentes. Aquí, los participantes que se han inscrito dialogan sobre diferentes aspectos y comparten sus experiencias con el fin de llegar a unas conclusiones generales. Por último, los progress reports son informes que envían algunos participantes y en donde se hace una exposición de la actividad de la institución a la que pertenecen. Estos documentos se cuelgan en la página web que se crea cada año y se comentan durante el seminario.

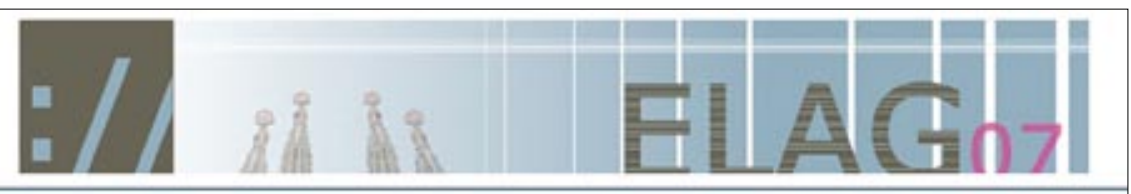

European Library Automation Group (ELAG)

Proceedings of the 31st Library System Seminar: ELAG 2007:

Library 2.0

University of barcelona, 9-11 May 2007

Edited by Pads Goossens

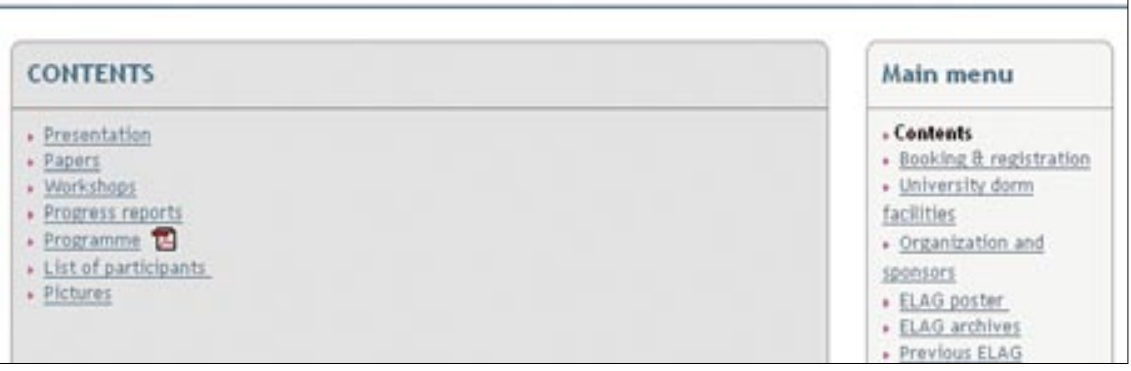

Página web del 31 th ELAG Seminar 


\section{"Los seminarios que organiza ELAG son una ocasión excepcional \\ para reunirse con especialistas de diferentes nacionalidades e intercambiar experiencias y opiniones"}

\section{Papers}

De los once papers presentados, tres tuvieron carácter local, con temáticas diversas: los consorcios de bibliotecas, los repositorios y el uso de las publicaciones periódicas en formato digital. Lluís Anglada, director del Consorci de Biblioteques Universitàries de Catalunya, en su exposición Twentyfive years of library automation in Catalonia, pasó revista al proceso de automatización que han seguido las bibliotecas catalanas en los últimos 25 años y presentó el estado actual de este proceso, incidiendo en la importancia de la cooperación entre bibliotecas y la creación de un único catálogo.

Reme Melero, del Instituto de Agroquímica y Tecnología de Alimentos de Valencia, se refirió a los depósitos digitales en acceso abierto en España en Open access institutional repositories: the case study of Spain. En su estudio destacó el rápido crecimiento de estos, particularmente entre los años 2004 y 2005, y la utilización de los programas Dspace y ePrints para implantarlos. En España, los depósitos digitales más frecuentes son institucionales y suelen contener, entre otras tipologías documentales, contribuciones a congresos, tesis, trabajos de licenciatura, pre-prints y post prints.

El último día Àngel Borrego, profesor de la Facultat de Biblioteconomia i Documentació de la Universitat de Barcelona, presentó las

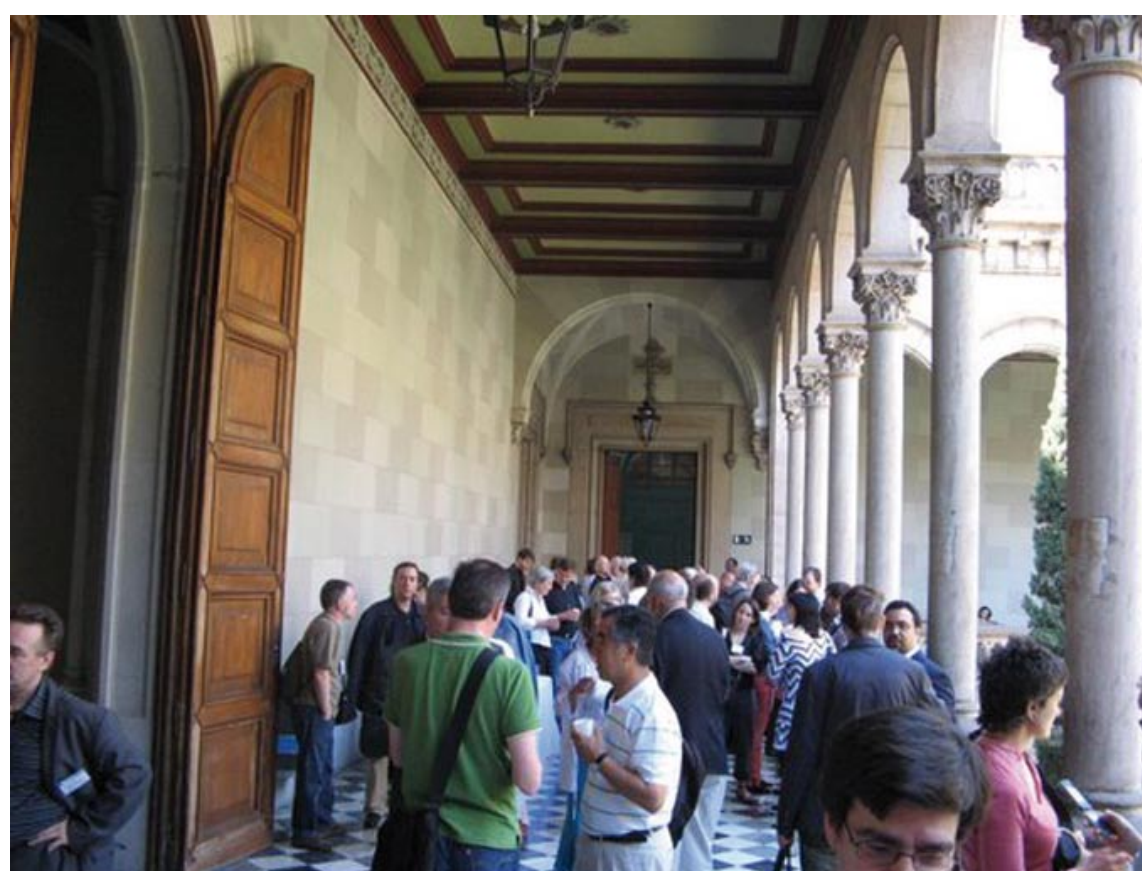

Asistentes al seminario. Foto Ramon Ros

conclusiones obtenidas a través de diversos estudios realizados sobre el uso de las revistas digitales en las universidades catalanas (E-journal usage studies at Catalan academic libraries).

De los papers de carácter general podemos destacar especialmente dos, el de Maja Zumer, de la Universidad de Ljubljana, y el de Herbert Van de Sompel, investigador de Los Alamos National Laboratory Research Library. Zumer presentó una conferencia que relacionaba Amazon y los catálogos de bibliotecas: Amazon: competition or complement to OPACs. Explicó como diversos estudios muestran las dificultades que tienen muchos usuarios para utilizar los catálogos de bibliotecas, debido especialmente a que las interficies de búsqueda son muy poco amigables. Esto provoca que algunos utilicen por ejemplo Amazon para buscar el ISBN y se sirvan de esta información para completar la búsqueda en el Opac. Zumer destacó las ventajas de la utilización del conocido portal de libros frente a los catálogos tradicionales: ofrece una interficie de búsqueda simple, muestra la cubierta del documento, permite ha- cer recomendaciones a los usuarios y ofrece la posibilidad, si existe, de enlazar con el texto completo. $\mathrm{Si}$ bien es cierto que algunas bibliotecas ya han empezado a aplicar algunas de estas funciones, la mayoría de centros siguen esperando para adaptar sus catálogos a las necesidades de sus usuarios. Tal y como insistió la ponente, sería necesario dirigir los cambios poco a poco en esa dirección y perder el miedo a equivocarse.

Van de Sompel centró su exposición en la descripción de los trabajos de $O R E$, el nuevo proyecto del equipo de Open Archive Initiative (OAI Object Re-Use and Exchange: Moving interoperability from the metadata to the resource level). ORE surgió a raíz del rápido aumento de repositorios, especialmente académicos, $\mathrm{y}$ de la gran cantidad de documentos que contienen. La idea de aprovechar estos repositorios y la documentación que hay en ellos hizo que el equipo de $O A I$ se planteara la posibilidad de aprovechar esta situación para crear una marco de comunicación digital internacional de documentos. Este marco facilitaría el flujo de los objetos que contienen los repositorios 


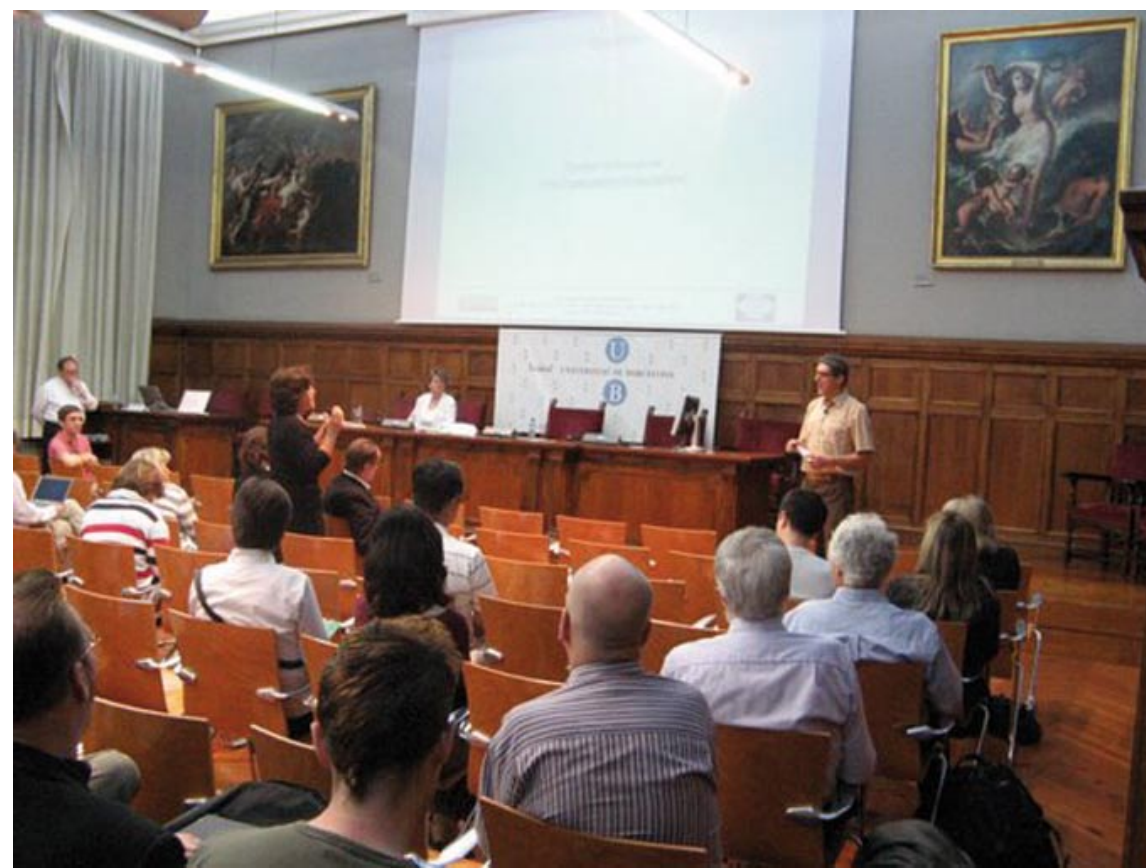

Van de Sompel durante su exposición. Foto Ramon Ros

y las diferentes partes de éstos (texto, imágenes, etc.). Así, se trata de crear un protocolo que permita no sólo intercambiar los metadatos de los documentos que contienen los repositorios sino intercambiar los mismos documentos y/o las diferentes partes de éstos.

El resto de papers, con autores de diferentes nacionalidades, trataron temas como: Moving towards a service-oriented architecture (Erlend Gutteberg), Electronic publishing and institutional archives: utilising open-source software (Ellen Royneberg), Accessing library material via Google and other websites (Janifer Gatenby), RDA (Resource Description and Access) (Gordon Dunsire), A futuristic view of knowledge and information management (Sue Mcnight) y The DELOS reference model for digital libraries (Vittore Casarosa).

\section{Workshops y progress reports}

Este año se ofreció a cada participante la posibilidad de participar en un workshop, en función de su especialidad y sus intereses. Los temas tratados en estos talleres fueron asimismo variados. Google y
La preservación también tuvo cabida en el Seminar de este año en el taller Preservation of digital content donde se habló de los estándares utilizados (MIX, PRE$M I S, O A I S, P D F / A)$, en la calidad, el control de la digitalización y las normativas existentes.

Los workshops con más participantes fueron los relacionados con sistemas y servicios 2.0, como: "Blogs, wikis,..." у "Library 2.0: what is in a name?". En el primero, coordinado por Ramon Ros, del Consorci de Biblioteques Universitàries de Catalunya, asistieron gran parte de los asistentes nacionales y se trataron temas relacionados con la aplicación en bibliotecas de algunas de las herramientas y tecnologías relacionadas con la web 2.0.

Por su parte, Ole Husby de Bibsys, el sistema de automatización de bibliotecas de Noruega, se encargó de coordinar el taller sobre el tema principal del Seminar, la Library 2.0. Los participantes en este grupo de trabajo profundizaron en ese concepto y plantearon algunas cuestiones que dejaron abiertas para posteriores reflexiones. Así, surgieron discusiones como la con-

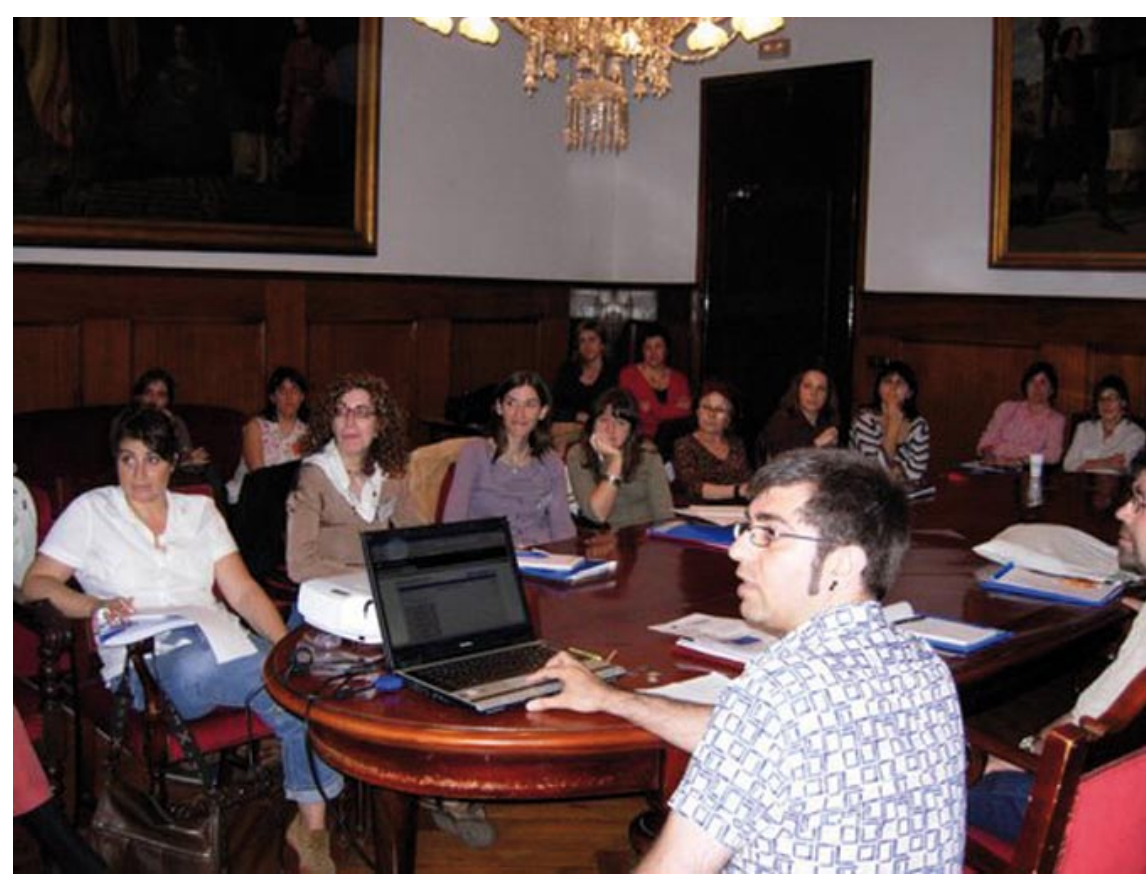

Workshop Blogs, wikis,... Foto Ramon Ros 


\section{El grupo ELAG}

ELAG (European Library Automation Group) es una organización formada por profesionales europeos interesados en las aplicaciones de las tecnologías de la información y la comunicación (TIC) en las bibliotecas.

Organiza desde hace más de 30 años seminarios anuales, que tradicionalmente se han caracterizado por tratar temas relacionados con las tecnologías en centros bibliotecarios, aunque últimamente la temática se ha diversificado más y se han explorado nuevos ámbitos como la web semántica, el open access, la interoperabilidad de sistemas o las nuevas herramientas bibliotecarias.

fianza que deben tener los profesionales en los usuarios para permitirles participar o la definición de los agentes implicados. Para profundizar en los temas tratados el coordinador del grupo diseñó un blog ${ }^{1}$ que utilizó como herramienta de trabajo y de comunicación en las diferentes sesiones y en la presentación general de los resultados que se hizo al final.

En el resto de workshops se trabajaron otros temas como la utilización de etiquetas o tags (Social tagging/indexing (User participation)), el acceso abierto (Opensource software: pros and cons) o el E-learning (E-learning and its effects on libraries).

Por su parte, los progress reports fueron presentados por Ramon Ros $\mathrm{y}$, aunque hay que lamentar que no hubiera demasiada participación, éste se encargó de realizar algunas preguntas a los responsables de los informes que sirvieron para generar diálogo y discusión.

\section{Conclusiones}

Los seminarios que organiza anualmente el grupo ELAG son una ocasión excepcional para reunirse con especialistas de diferentes na- cionalidades e intercambiar experiencias y opiniones. Las distintas ubicaciones geográficas del seminario permiten a los asistentes conocer, mediante los papers locales, contextos bibliotecarios y experiencias que, de otro modo, difícilmente hubieran conocido.

Después de escuchar las diferentes aportaciones de los papers, los progress reports y los workshops, una de las cuestiones que cobra más fuerza es que la biblioteca 2.0 ofrece a los profesionales y a las instituciones a las que pertenecen una gran oportunidad para mejorar sus servicios y su reconocimiento. Aunque actualmente ya hay diversas bibliotecas que empiezan a utilizar herramientas características de la web 2.0, como los blogs, en este Seminar se han señalado algunos aspectos cruciales que están frenando su avance como son la falta de confianza en el usuario y el miedo a la manipulación externa del catálogo.

Es necesario por tanto que los profesionales empecemos a adaptarnos a este nuevo contexto, confiando en los usuarios y en sus aportaciones y planteándonos la posibilidad de cambiar y de arries-

\author{
"Es necesario que los \\ profesionales empecemos \\ a adaptarnos a este nuevo \\ contexto, confiando en \\ los usuarios y en sus \\ aportaciones"
}

garnos con nuevas propuestas, ya que, como apostilló Maja Zumer: "It is better to make an occasional wrong step than stand still and disappear into disuse”.

Para profundizar en los temas tratados en este seminario, se puede consultar desde mediados de octubre en los Proceedings of the 31st Library Systems Seminar: ELAG 2007: Library 2.0 ${ }^{2}$ toda la documentación generada referente a los papers, workshops y progress reports. Asimismo, también se pueden ver fotografías del acontecimiento y consultar otras informaciones de interés.

Por último, se puede anunciar ya que el próximo ELAG Seminar, el número 32, tendrá lugar entre los días 14 y 16 de abril de 2008 en el University and Research Centre Library de Wageningen, (Holanda), con el lema central "Rethinking the Library". Actualmente ya se puede consultar el programa en su web ${ }^{3}$, por lo que es un buen momento para empezar a preparar nuestras agendas y reservarnos esos días para la asistencia al próximo Seminar donde tendremos ocasión de replantearnos una nueva biblioteca.

\section{Referencias}

1. http://ws5e.wordpress.com/

2. http://elag2007.upf.edu/

3. http://library.wur.nl/elag2008/

Sílvia Redondo, Universitat de Barcelona, Facultat de Biblioteconomia i Documentació redondosil@gmail.com 